\title{
1 Disease mediated changes to life 2 history and demography threaten the 3 survival of European amphibian 4 populations
}

\section{Authors}

6 Lewis J. Campbell ${ }^{1,2}$, Trenton W. J. Garner ${ }^{2}$, Giulia Tessa ${ }^{3}$, Benjamin C. Scheele ${ }^{4}$, Amber G.F.

7 Griffiths $^{5}$, Lena Wilfert ${ }^{6}$, Xavier A. Harrison ${ }^{2}$.

\section{Affiliations.}

9 1.Environment and Sustainability Institute, University of Exeter, Penryn Campus, Penryn, 10 Cornwall, TR11 9FE, U.K

11 2.Institute of Zoology, Zoological Society of London, Regents Park, London, NW1 4RY, U.K

12 3. Life science and Systems Biology Department, Università degli Studi di Torino, via

13 Academia Albertina 13, 10123, Torino, Italy

14 4. Fenner School of Environment and Society, Australian National University, Canberra, ACT

152601 , Australia

16 5. FoAM Kernow, Studio E, Jubilee Warehouse, Commercial Road, Penryn, Cornwall TR10 8FG

17 6. Centre for Ecology and Conservation, University of Exeter, Penryn Campus, Penryn,

18 Cornwall, TR11 9FE 


\section{Abstract}

23 Infectious diseases can influence the life history strategy of their hosts and such influences

24 subsequently impact the demography of infected populations, reducing viability

25 independently of increased mortality or morbidity. Amphibians are the most threatened

26 group of vertebrates and emerging infectious diseases play a large role in their population

27 declines. Viruses of genus Ranavirus are responsible for one of the deadliest of these

28 diseases. To date no work has evaluated the impact of ranaviruses on host life-history post

29 metamorphosis or population demographic structure at the individual level. In this study, we

30 used skeletochronology and morphology to evaluate the impact of ranaviruses on the

31 demography of populations of European common frog (Rana temporaria) in the United

32 Kingdom. We compared ecologically similar populations that differed only in their historical

33 presence or absence of ranaviral disease. Our results suggest that ranaviruses are associated

34 with shifts in the age structure of infected populations, potentially caused by increased adult

35 mortality and associated shifts in the life history of younger age classes. Population

36 projection models indicate that such age truncation could heighten the vulnerability of frog

37 populations to stochastic environmental challenges. Our individual level data provide further

38 compelling evidence that the emergence of infectious diseases can alter host demography,

39 subsequently increasing population vulnerability to additional stressors. 


\section{Introduction}

Infectious diseases can have major long-term impacts on host life history strategies, often leading to subsequent shifts in the demographic structure of host populations [1-4]. Within age-structured populations, this is primarily caused by compensatory changes in the vital rates (rates of growth, fecundity and survival) of younger age classes that occur in response to high levels of extrinsic adult mortality (death of adult animals attributable to external factors such as disease or predation $[5,6])$. In order to maximise individual fitness within an environment of high extrinsic adult mortality, selection for increased juvenile survival and developmental rates, decreased size and age at sexual maturity and ultimately an increased adult life span (deceased intrinsic adult mortality) will occur [7]. This has been empirically borne out in a number of systems in response to several sources of mortality including; predation [8], over-harvesting [9] and experimentally induced adult mortality [7]. Impacts on population demographic structure potentially brought about by changes to individual life history strategies can have substantial effects on the growth and stability of infected populations [10]. This has recently been well demonstrated by Scheele et al. [11] who observed that infection with the lethal fungal pathogen Batrachochytrium dendrobatidis $(B d)$ causes high levels of adult mortality in the endangered Australian alpine tree frog (Litoria verreauxii alpina). High adult mortality resulted in a severe truncation of the age structure of infected populations. Subsequent population projection modelling showed that such $B d$ positive populations are much more vulnerable to decline due to stochastic recruitment failure caused by a variable environment than $B d$ negative populations [11], highlighting an important, yet relatively unexplored mechanism by which infectious diseases can impact their hosts. Infectious diseases are emerging at a faster rate and threatening a larger range of species than at any prior point in history [12], therefore it is imperative that we aim to better understand such mechanisms, particularly in species of conservation concern. 

such as habitat loss [14], over-harvesting [15] and climate change [16], one major driver of continued amphibian declines is the emergence of a suite of infectious diseases [17]. One of the most widespread and deadly of these diseases, ranavirosis, is caused by viral pathogens belonging to the genus Ranavirus $[18,19]$. Ranaviruses are globally distributed and infect and

71 kill species from 3 classes of ectothermic vertebrates [20-22]. Clinical ranavirosis is often characterised by severe dermal ulcerations as well as haemorrhaging and lesions affecting the internal organs $[22,23]$.Outbreaks are known to cause mass mortality events, with up to within a population can potentially lead to greater than $80 \%$ declines in population sizes, followed by suppression or in some cases local extinction [24]. Susceptibility varies among species but a number of ecological risk factors that contribute to species susceptibility, including life history strategy, have been identified [25]. The presence of ranaviruses within

79 a habitat has also been shown to accelerate the developmental rates of larval North American anurans [26]. To date no work has evaluated the impact of ranaviruses on life history strategies post metamorphosis, or on the demographic make-up of populations using individual level data. In this study, we utilised the unique comparative field system borne out of the Frog Mortality Project (FMP; see $[24,27]$ for details) to study the impacts of ranaviral disease history on demographic structure and individual life history of European common frog $(R$. temporaria) in the United Kingdom. We used a combination of skeletochronology (age

87 determination by skeletal growth rings) and morphometric data collected from wild caught

88 R. temporaria to test two hypotheses: i) that the age structure of populations with a positive 89 disease history of ranavirus will be truncated and ii) that frogs originating from ranavirus 90 positive field sites will display reduced body size and more rapid growth compared to their 91 counterparts from ostensibly disease-free populations. Additionally, we performed 
population matrix modelling to investigate whether persistent ranavirosis heightens

93 population susceptibility to additional external stressors.

\section{Methods}

\section{Field Sampling}

96 Potential field sites were drawn from the FMP database of $R$. temporaria populations known

97 to have experienced at least one mass mortality event due to ranavirosis and a

98 complimentary database of putatively disease free $R$. temporaria populations (see [24] for

99 more detailed selection criteria). Five populations of each disease history were selected (Fig

100 1). Field sites were attended during the spring breeding season and each was sampled for

101 one day. Sampling involved searching for frogs within and around the proximity of the

102 breeding ponds. As many individuals as possible, including any amplexing pairs, were located

103 and placed into plastic holding tanks (care was taken not to cause paired frogs to release).

104 Since as many frogs as possible were captured at each field site by the same individual

105 researcher, sampling effort is considered to be approximately equal. As all frogs were found

106 to be above the age minimum age of sexual maturity, and juvenile anurans rarely return to

107 breeding ponds [28,29 but see 30$]$, all were assumed to be members of the breeding

108 population.

109 Snout to vent length was measured using $0.1 \mathrm{~mm}$ scale callipers. For unpaired frogs,

110 body weight was recorded using a 100g maximum weight (1g increments) Pesola drop scale.

111 Frogs were weighed in a plastic zip lock bag of known weight (4g), which was subtracted

112 from the total reading of the scale. A clean zip lock bag was used for each individual.

113 The distal portion of the $1^{\text {st }}$ (inside) digit of a hind limb of each frog was clipped

114 using surgical scissors. To minimise the potential for pain and the possibility of infection, a

115 topical disinfectant that contained an analgesic (Bactine; WellSpring Pharmaceutical,

116 Sarasota, USA) was applied to the surgical area prior to the procedure. Toe clips were placed 
117 into individual $1.5 \mathrm{ml}$ micro-centrifuge tubes containing $1 \mathrm{ml}$ of $70 \%$ ethanol. Following

118 sampling, all animals were released back into the breeding ponds. The number of individuals

119 sampled per each population varied between 4 (Witham) and 61 (Mitcham and Palmer's

120 Green) with a mean of 30 animals sampled per site (Supplemental table S1.).

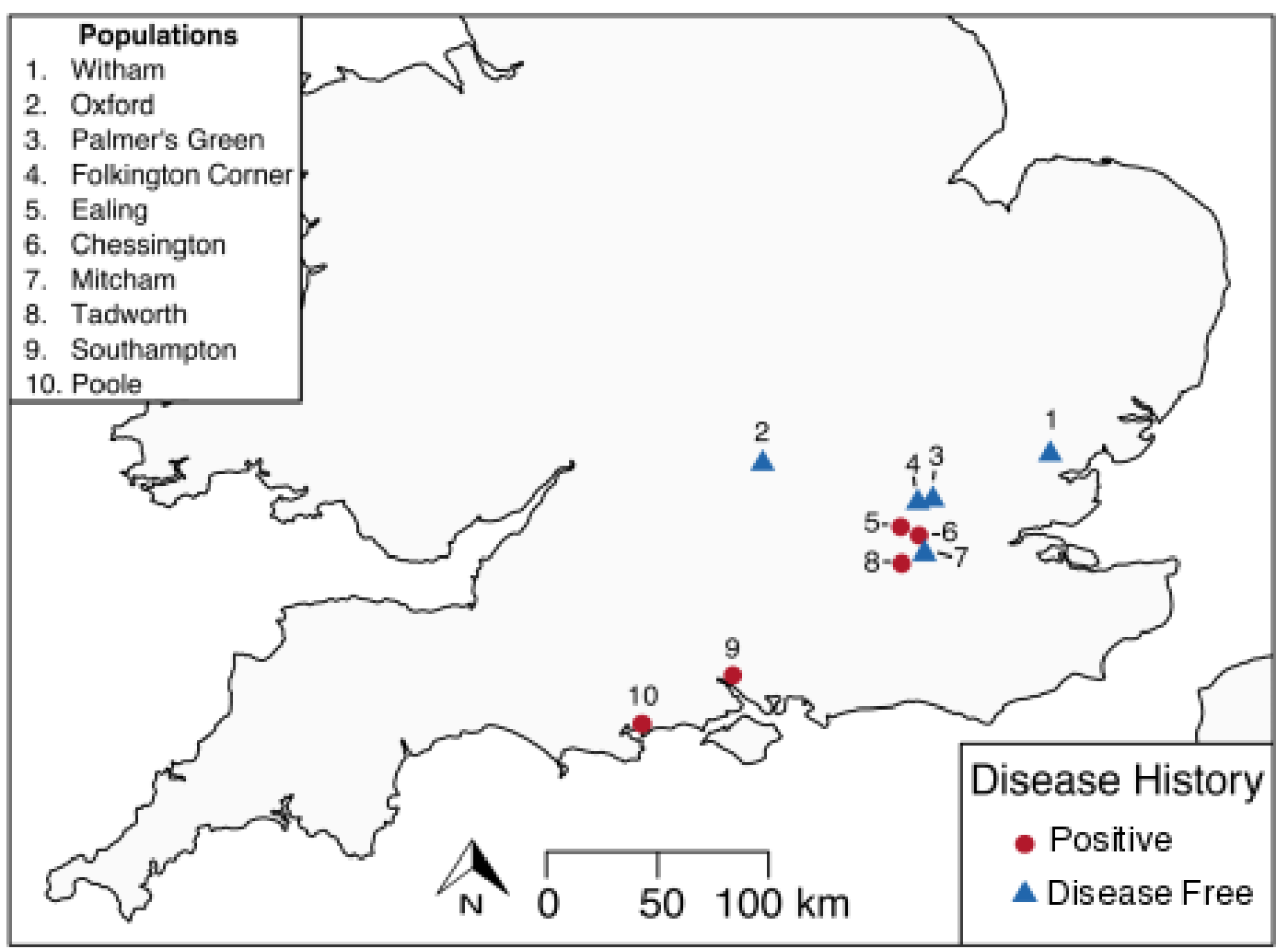

Fig 1 Map of the locations of sampled populations within the southern United Kingdom. Field sites were drawn from the Frog Mortality Project database of populations known to have experienced FV3 mass mortality events and a complimentary database of populations known to have been FV3 free since disease emergence in the early 1990s.

\section{Age determination}

122 Age was determined by skeletochronology, following the protocol for $R$. temporaria 123 published by Miaud et al [31] with the following modifications. The phalangeal bone was 124 separated from soft tissues, decalcified with $5 \%$ nitric acid for 1.5 hours and washed with 125 water over night. Cross sections ( $12 \mu \mathrm{m}$ thick) were then cut from the bone using a cryostat 
126 and stained using haematoxylin for 20 minutes. Lines of arrested growth (LAG) were

127 counted using a light microscope at 200x-400x magnification, 10-12 sections were analysed

128 for each individual and two different researchers verified counts. Age at sexual maturity was

129 determined as the youngest age at which inter-LAG space reduced in size, as juvenile inter-

130 LAG space is significantly wider than post sexual maturity [32].

\section{Statistical Analyses}

\section{Body size by age and age at sexual maturity.}

133 We conducted all statistical modelling in $\mathrm{R}$ [33]. We used linear mixed effects regression

134 (Imer) models, implemented in the package Ime4 [34] fitted with a Gaussian error structure,

135 and a stepwise simplification procedure to investigate the impact of population ranaviral 136 disease history on the body size (SVL) of $R$. temporaria. Sex, ranaviral disease history of the 137 source population and their interactions were fitted as fixed effects and source population 138 alone as a random effect (Table 1). Since male and female frogs grow at different rates $139[31,35,36]$ the datasets of each sex were analysed separately.

A separate Imer model was fitted to investigate the impact of the ranavirus history

141 status of the source population on age at sexual maturity. In the full model, age at maturity 142 was fitted as the response variable, ranaviral disease history of source population as a fixed 143 effect and source population as a random effect. As female $R$. temporaria mature later than 144 males $[31,36]$ the datasets of each sex were analysed separately. population as a random effect. We used uninformative priors for both the random effect (G)

151 and residual variance $(R)$ structures, but fixed the residual variance at 1 as this quantity 
152 cannot be estimated in ordinal models. The model was run for a total of 600000 iterations

153 with a burn-in period of 100000 iterations and a thinning rate of 500 , giving a final sample of

1541000 draws from the posterior distributions. Mean probability of membership and $95 \%$

155 credible intervals for each age class for each of the two disease history groups was

156 calculated from the linear predictor. We assessed model convergence using the Gelman-

157 Rubin statistic calculated from two independent chains initiated with overdispersed starting

158 values. All G-R values were $<1.05$, indication convergence (see supplementary figure S9 for

159 diagnostic plots). Age structure plots (Fig 3) suggest that observed changes were similar for

160 both sexes, so to increase power the dataset was not split by sex.

\section{Population Matrix Modelling}

163 Initial matrix construction and population projections

164 To investigate the potential for changes in demography to impact the long-term viability of

165 R. temporaria populations, we conducted population matrix modelling. An initial female-

166 based Leftkovich matrix was produced consisting of 11 stages; eggs, juvenile (sexually

167 immature frogs) and adult age classes ranging from 2 to 10 years old. Leftkovich matrices

168 are stage based and split the female (providing females are the limiting sex) component of a

169 population into classes based on age, size, developmental stage or similar depending on the

170 study species. The survival of each age class into the subsequent age class is represented as

171 a proportion and the fecundity of each class into every other class (if applicable) as a raw

172 value of females generated per female. See supplementary material for structural examples

173 of Leftkovich matrices. Our basal matrix, representing a putatively disease-free population,

174 was constructed using published vital rates for $R$. temporaria from Biek et al. [38]. Biek et al

175 assume a constant and uniform maximum reproductive output for all sexually mature adult

176 females of 650 eggs per year. However, it has been shown that the reproductive output of $R$.

177 temporaria is thought to be significantly influenced by body size [38 but 39]. In conjunction 
178 with the body size by age data generated in our field study, we adjusted the vital rates for

179 fecundity to increase stepwise for every year post sexual maturity, starting at a rate of 250

180 eggs for two-year-old breeding animals and increasing by 50 eggs per year until an output of

181650 eggs per year was attained at adult age class 10. The rates of Biek et al. [38] also assume

182 transition from juveniles directly into adult class 2 . However, since fecundity in our matrix is

183 not uniform and female frogs from our dataset were found to mature at ages 2,3 and 4

184 (Supplementary Figure S8, consistent with previous findings in R. temporaria [31]), we

185 accounted for variation in onset of sexual maturity by allowing juveniles to remain juveniles

186 and to transition directly into adult classes 2,3 or 4 with highest chance of reaching maturity

187 at adult stage 3. See supplementary table S2 for a numerical representation of the full basal

188 matrix.

189 We created two age distribution vectors (one for each disease history group) by

190 calculating the proportion of individuals observed in populations of each disease history

191 type that represented each age class and using those proportions as probabilities by which

192 to weight a draw of 150 individual females that could belong to any age class. We used the

193 projection function of the Popdemo package [40] to project these starting populations 20

194 years into the future based on our starting matrices (additional parameters; standard. $A=$

195 True, standard $\cdot v e c=$ True $).$

196 Deaths due to ranavirosis are known to occur annually in diseased populations of UK

197 common frogs $[17,24]$ and annual breeding in permanent water bodies, within which

198 ranaviruses can persist [41], has been identified as a key ecological risk factor of disease

199 [25]. To represent an increased annual chance of an individual dying from ranavirosis every

200 year that it returns to an infected water body, we created additional matrices, which

201 reduced adult survival annually. No age-specific data are currently available on ranavirus

202 prevalence and mortality rates of wild adult amphibians caused by ranavirosis. We therefore

203 modelled the population dynamics of a range of percentage decreases in annual survival in 
204 positive disease history populations ( $1 \%-25 \%)$. We found no significant difference in

205 population dynamics of any starting population based on decreasing annual adult survival

206 alone (supplementary figure S3), we therefore selected a reasonable value of a 5\% increased

207 likelihood of mortality per year to represent theoretical populations affected by lethal

208 ranavirosis (Supplementary Figure S3).

\section{Stochastic population projections}

210 To investigate the potential impact of environmental stochasticity on populations

211 experiencing persistent disease (5 \% increased adult mortality per year of age) and those

212 that are not affected by ranavirosis we created an additional set of matrices to represent

213 differing ecological conditions. A well-known threat to the stability of amphibian populations

214 is climate induced reproductive failure [11,42], and in the UK fluctuations in late

215 winter/early spring night time temperatures can result in frost killing amphibian spawn. Low

216 water temperatures have been identified as the principle threat to $R$. temporaria spawn in

217 UK ponds [43] and are also associated with oomycete infections of amphibian egg masses,

218 which can result in total reproductive failure [44]. To examine the impact of such scenarios,

219 we created alternate versions of both starting population matrices in which fecundity of all

220 sexually mature adult classes was reduced to 0 (supplementary tables S4 and S5).

221 To examine the impact of potential recurrence of mass mortality events due to

222 ranavirosis, we created a further two matrices; one where the survival of all sexually mature

223 adult classes was reduced to $20 \%$, a biologically plausible but not extreme mortality

224 estimate given that greater than $90 \%$ mortality has been observed in mass mortality events

225 caused by ranavirosis [19] and a final matrix representing a catastrophic year in which both

226 adult mass mortality and total reproductive failure occurred (post-maturity adult survival of

$22720 \%$ and fecundity of 0 , supplementary tables S6 and S7.) We used the stochastic projection

228 function of the pop.bio package [45] to run the following stochastic projections;

229 A) Disease-free population with a $10 \%$ annual chance of reproductive failure; 
B) Population affected by lethal ranavirosis with a $10 \%$ annual chance of reproductive failure;

C) Population affected by lethal ranavirosis with a $10 \%$ annual chance of either reproductive failure or an adult mass mortality event;

D) Population affected by lethal ranavirosis with a $10 \%$ annual chance of either reproductive failure or an adult mass mortality event and a $5 \%$ annual chance of both challenges occurring simultaneously.

To ensure all stochastic projection populations started equally, we used the starting population vector of the disease-free populations for all stochastic projections. All projections were run for 100 years and for 5000 iterations. Based on initial population 240 projections we enforced a ceiling carrying capacity for our theoretical populations of 200 241 sexually mature adult females summed across all age class.

\section{Results}

\section{Body size by age and age at sexual maturity}

244 We sampled 208 male and 66 female frogs, of which 103 males and 31 females were

245 sampled at populations where lethal ranavirosis had been reported.

246 For both male and female sub groups, age had a highly significant effect on SVL

247 (males; $\mathrm{df}=4$, Chisq $=186.38, \mathrm{p}<0.001$, females; $\mathrm{df}=4$, Chisq $=47.16, \mathrm{p}<0.001 ;$ Fig. 2; Table

248 1). Frogs from positive disease history sites were larger per age class, but this was a non-

249 significant effect (Table 1). Mean age at sexual maturity of males from diseased field sites $250(n=57)$ was 2.6 years $( \pm$ SE 0.07$)$ and 2.8 years ( \pm SE 0.06$)$ at locations where disease was not 251 reported $(n=59)$. Mean age at sexual maturity of females from positive field sites $(n=19)$ was 2523.2 years $( \pm$ SE 0.13$)$ and for females from negative populations $(n=17)$ it was 3.3 years $( \pm$ SE 253 0.10), and all differences were non-significant (Supplementary Figure S8; Table 1.) 
bioRxiv preprint doi: https://doi.org/10.1101/178723; this version posted August 20, 2017. The copyright holder for this preprint (which was

not certified by peer review) is the author/funder, who has granted bioRxiv a license to display the preprint in perpetuity. It is made available under aCC-BY-ND 4.0 International license.

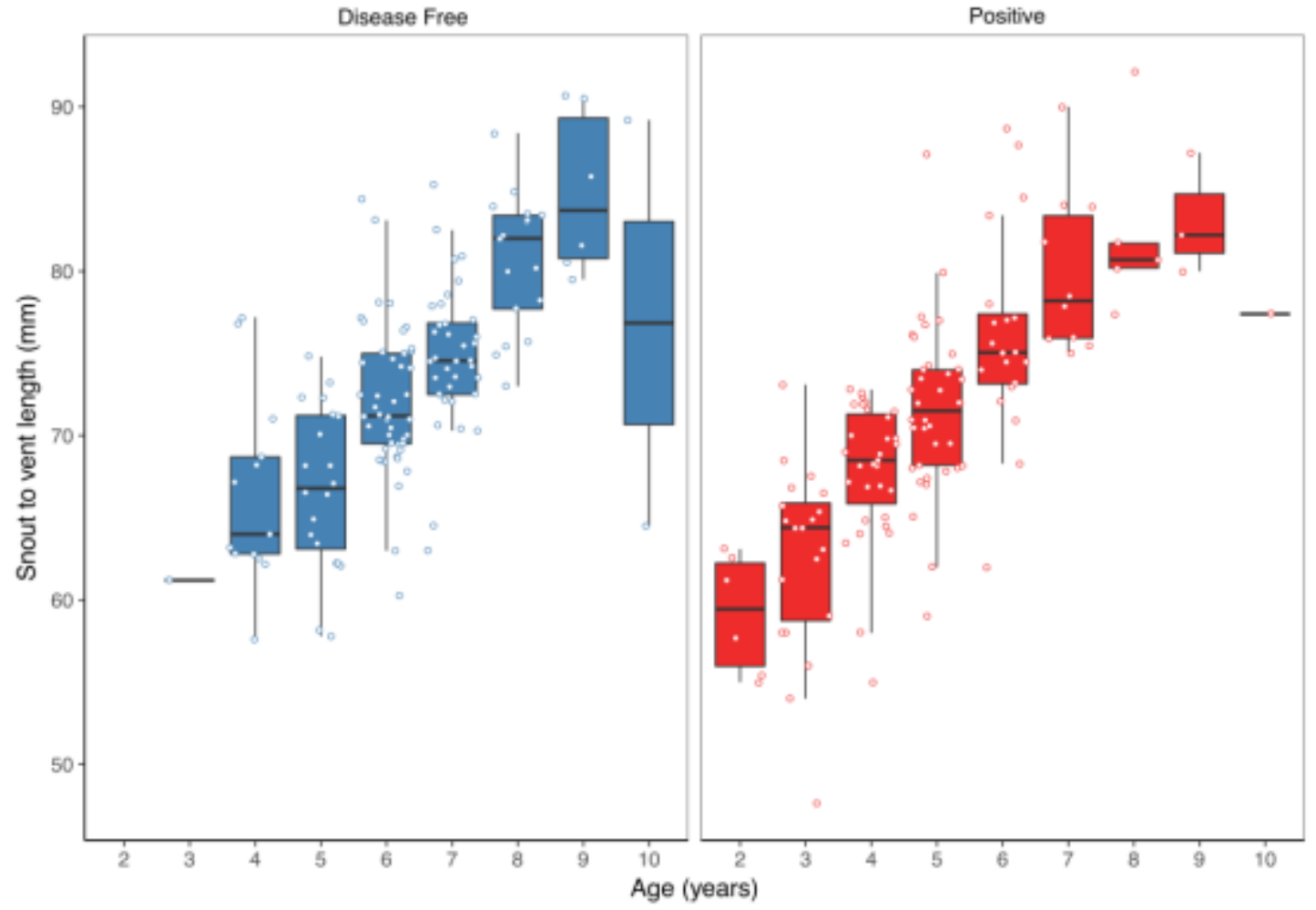

Fig 2 - The relationship between snout to vent length (SVL) and age in $R$. temporaria from ranavirus positive and disease free populations. We found no significant effect of disease history on the SVL of frogs. Our data does however show clear evidence of continued growth throughout life regardless of disease history. 


\begin{tabular}{|c|c|c|c|c|}
\hline \multicolumn{5}{|c|}{ Male Body Size } \\
\hline Fixed effects structure & Removed fixed effect & df & $\mathrm{Chi}^{2}$ & $p$ \\
\hline svl age * status & & 6 & & \\
\hline svl age + status & Age * Status & 5 & 0.0587 & 0.8085 \\
\hline svl age & Status & 4 & 0.21 & 0.6467 \\
\hline$\left.s v\right|^{\sim 1}$ & Age & 3 & 186.38 & $<0.001$ \\
\hline \multicolumn{5}{|c|}{ Female Body Size } \\
\hline Fixed effects structure & Removed fixed effect & df & $\mathrm{Chi}^{2}$ & $p$ \\
\hline svl age * status & & 6 & & \\
\hline svl age + status & Age * Status & 5 & 0.9383 & 0.3327 \\
\hline svl age & Status & 4 & 2.4276 & 0.1192 \\
\hline svl 1 & Age & 3 & 47.16 & $<0.001$ \\
\hline \multicolumn{5}{|c|}{ Male age at maturity } \\
\hline Fixed effects structure & Removed fixed effect & df & $\mathrm{Chi}^{2}$ & $p$ \\
\hline agemat $\sim$ status & & 4 & & \\
\hline agemat $\sim 1$ & Status & 3 & 0.9869 & 0.3205 \\
\hline \multicolumn{5}{|c|}{ Female age at maturity } \\
\hline Fixed effects structure & Removed fixed effect & df & $\mathrm{Chi}^{2}$ & $p$ \\
\hline agemat $\sim$ status & & 4 & & \\
\hline agemat 1 & Status & 3 & 0.2969 & 0.5858 \\
\hline
\end{tabular}

Table 1 Model summaries of model simplification procedure to evaluate the effect of ranaviral disease history on the body size and age at maturity of $R$. temporaria populations. All models contained only population of origin as a random effect. The $p$ values presented here represent the significance of the parameter removed from the preceding model as calculated by a likelihood ratio test between models (anova in R.) svl = snout to vent length, agemat = age at sexual maturity and $d f=$ degrees of freedom of the model. 


\section{Influence of Ranavirus on population age structure.}

259 The mean age of males from populations where disease related mortality had been reported

260 was 4.8 years old ( \pm SE 0.16 ) compared to an average age of 6.3 years ( \pm SE 0.13 ) at sites

261 where disease has not been reported. Mean female age at diseased sites was 5.3 years $( \pm$ SE

2620.28 ) compared to 6.6 years ( \pm SE 0.26 ) at locations where only healthy frogs had been

263 observed.

264 Disease history had a significant effect on age structure (effect size -1.43 , lower $95 \%$

265 credible interval -2.37, upper $95 \%$ credible interval $-0.38, p=0.008$; Fig 3), showing

266 populations with a positive history of ranavirosis to be dominated by younger $R$. temporaria.

267 We calculated the difference in posterior probabilities of belonging to an age class based

268 upon disease history status by subtracting the posterior probability of a frog of age $\mathrm{X}$ being

269 encountered at positive history population from the posterior probability of a frog of age $X$

270 being found at a disease-free population. The resulting difference values show that adults

271 aged 2 - 5 years old are more likely to be encountered in disease positive populations, and

272 those aged $6-10$ years old are more likely to be observed at populations where no disease

273 has been recorded (Fig 4.) Differences in age distributions were strongly supported for all

274 age classes (95\% credible intervals (Cl) of difference do not cross zero), except for six year

275 olds. Although six-year-old frogs were more likely to be observed in populations where

276 disease had not been reported, the $95 \%$ credible intervals incorporated 0 (mean difference $=$

$277-14.41,95 \% \mathrm{Cl} \quad-30.45$ to $3.96 ;$ Fig 4). 

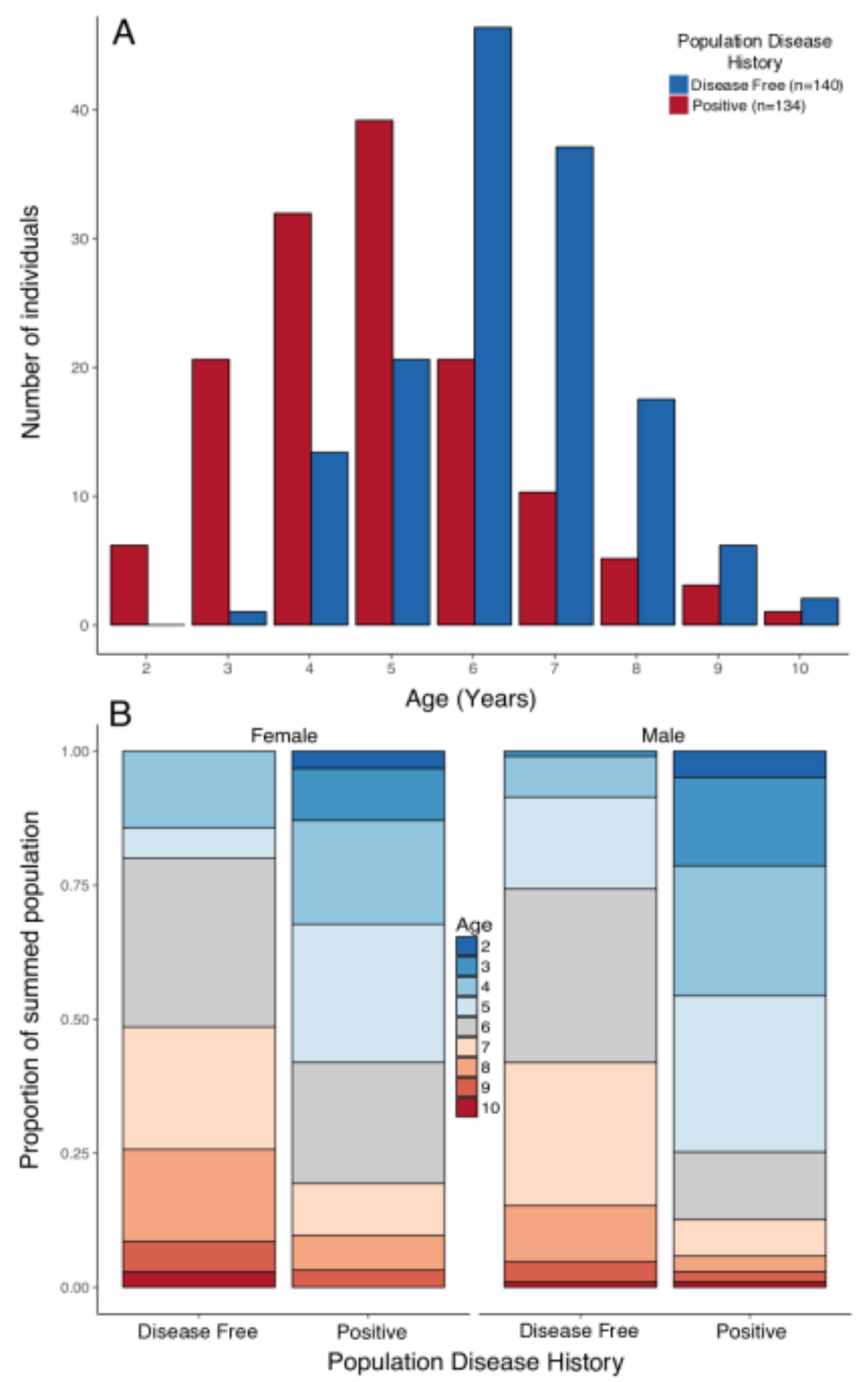

Fig 3 - A) Histogram of raw counts of numbers of individuals observed per age class per disease history status type. B) Proportional stacked bar chart of the proportion of individuals found in populations of each disease history that was a given age, broken down by sex. Breeding populations with a poisitive disease history of ranavirus are dominated by animals 5 years of age and younger. Populations with no history disease are majorly comprised of animals 6 years of age and older. 


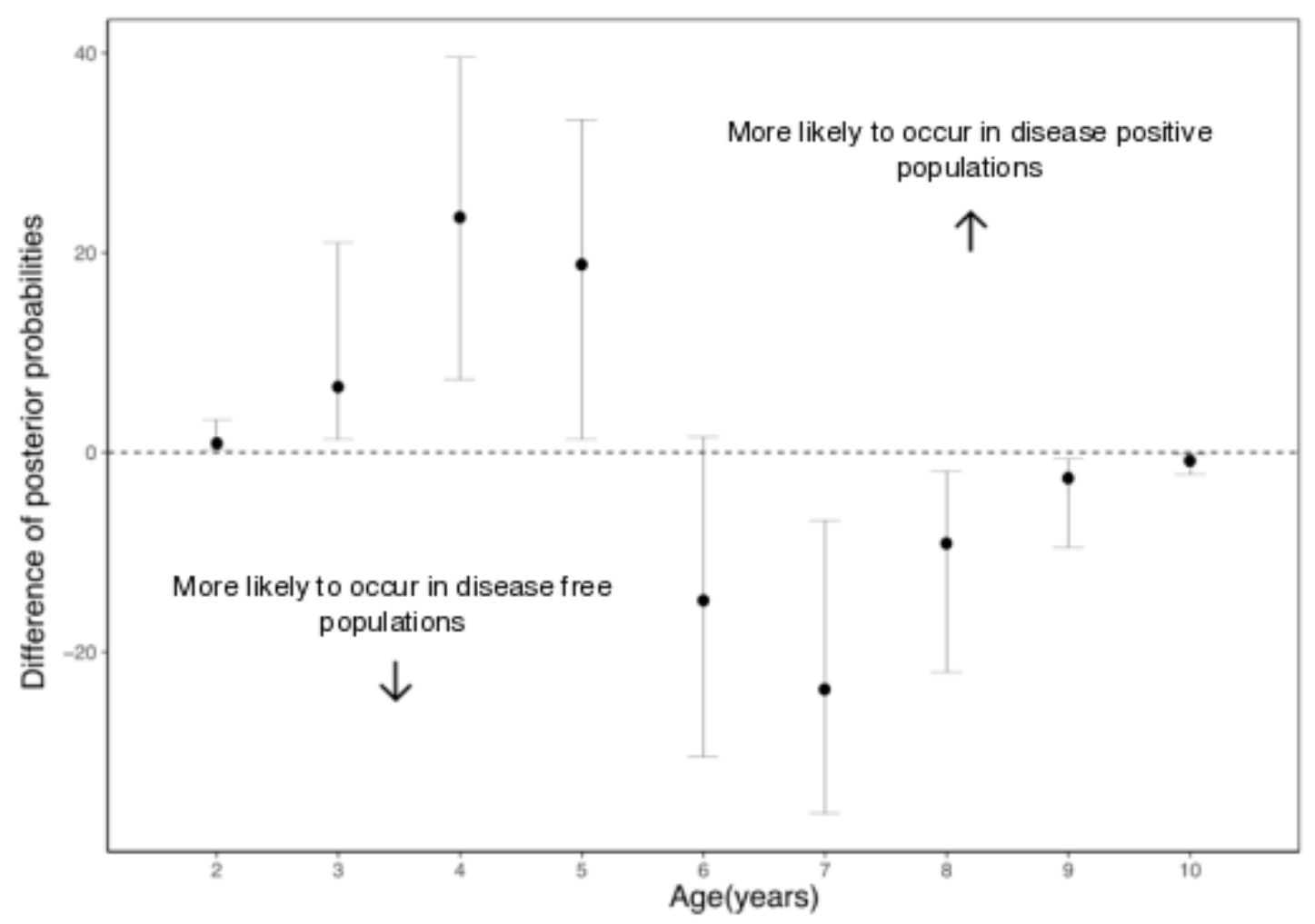

Fig 4 - The mean difference in the posterior probabilities of belonging to a given age class by population infection history status. Values $>0$ indicate that an age class is more likely to be observed in a positive disease history population and $<0$ a putatively disease free population. An age with $95 \%$ $(2.5 \%-97.5 \%)$ credible intervals that do not span zero suggests that influence of disease status on that age class is supported by our model. This is the case for all classes other than age 6 which although found to be observed more often in disease free populations has credible intervals spanning 0. 


\section{Population projection modelling}

283 Initial projections of the population dynamics of positive (5\% reduction in adult survival per

284 year of age) and disease free populations showed that both followed approximately equal

285 trajectories. Population growth initially spiked due to an influx of pre-mature age classes

286 into the population, followed by a period of attenuating oscillation before reaching

287 equilibrium at around the fifteenth year (Supplementary Figure S10). Final sizes of disease

288 positive populations were non-significantly smaller (positive $n=\sim 146$ vs disease free $n=\sim$

289 182; $t$-test, $t=1.67, d f=39.76, p=0.102)$.

290 Disease free populations subject to a $10 \%$ chance of recruitment failure per year

291 were still able to consistently attain populations sizes near carrying capacity much more

292 often than any disease positive population (Fig 5, table 2). Increasing complexity of

293 stochastic scenarios reduced the viability of populations experiencing ranaviral disease.

294 Disease positive populations where both adult mass mortality and reproductive failure could

295 occur within the same year were the least viable, being driven locally extinct in $58 \%$ of

296 model iterations (Fig 5, table 2). 


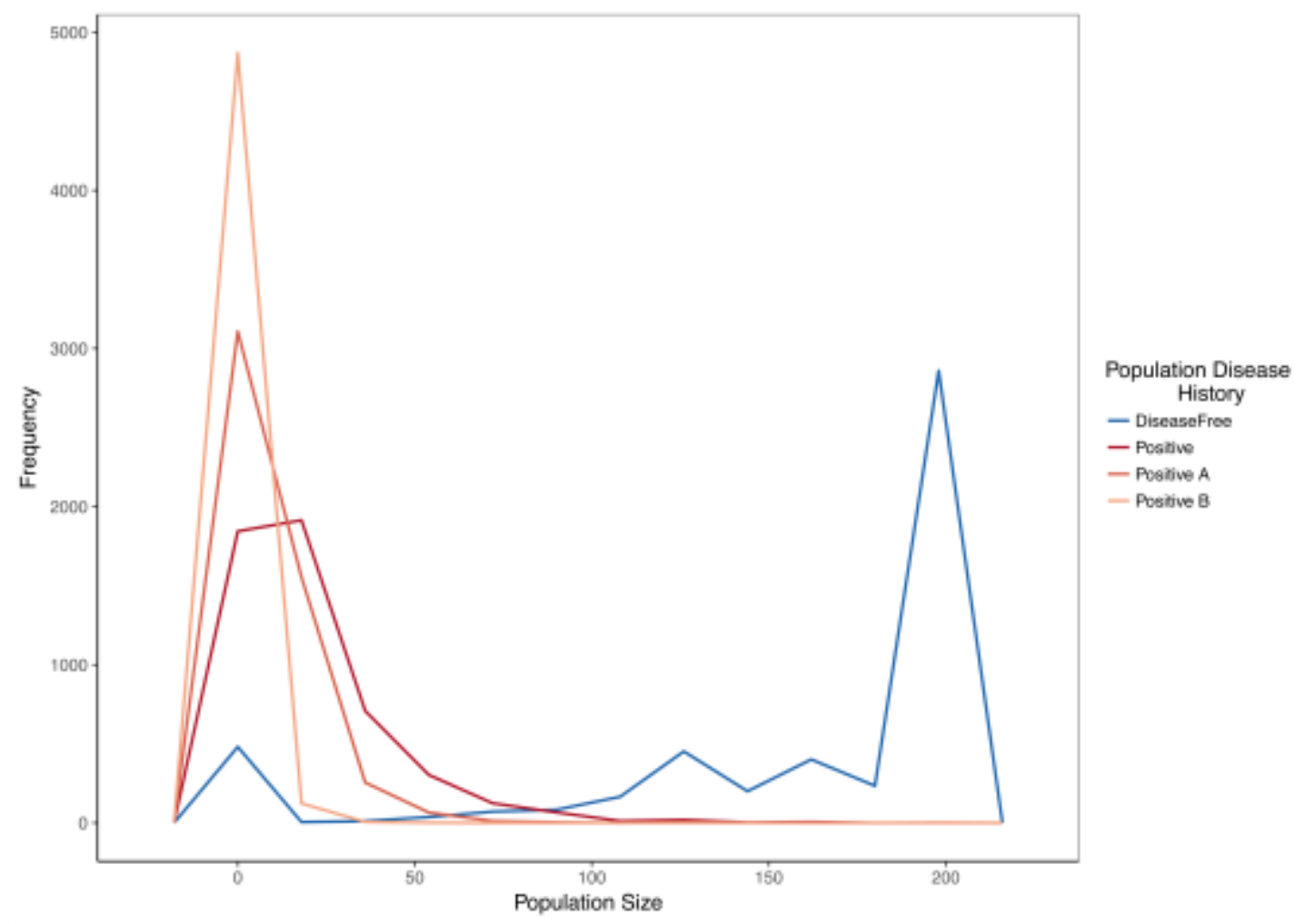

Fig 5 Frequency polygon of iterations in which the projected population hit a given size in stochastic projection modelling. The same starting population vector based on summed observed disease free populations was used in all models. Disease Free = Simulated disease free population under a $10 \%$ annual chance of complete reproductive failure. Positive $=$ Simulated positive disease history population under a $10 \%$ annual chance of complete reproductive failure. Positive $A=$ Simulated positive disease history population under a $10 \%$ annual chance of reproductive failure AND a $10 \%$ annual chance of a recurrent adult mass mortality event in exclusive years. Positive $B=$ Simulated positive disease history population under identical conditions to Positive A with addition of a $5 \%$ annual chance of complete recruitment failure and adult mass mortality in the same year. 


\begin{tabular}{|lcccccc|}
\hline \multicolumn{1}{|c}{ Model } & Extinct & $<\mathbf{5 0}$ & $\mathbf{5 0 - 1 0 0}$ & $\mathbf{1 0 0 - 1 5 0}$ & $\mathbf{1 5 0 - 1 9 9}$ & $\boldsymbol{K}$ \\
\hline Disease Free & 12 & 525 & 177 & $\mathbf{7 3 4}$ & 1319 & $\mathbf{2 2 3 3}$ \\
Positive & 429 & 4062 & 443 & 48 & 15 & 3 \\
Positive A & 611 & 4300 & 87 & 2 & 0 & 0 \\
Positive B & 2918 & 2081 & 1 & 0 & 0 & 0 \\
\hline
\end{tabular}

Table 2 The number of iterations per 5000 that each stochastic projection model reached a given population size. Disease Free = Simulated disease free population under a $10 \%$ annual chance of complete reproductive failure. Positive $=$ Simulated positive disease history population under a $10 \%$ annual chance of complete reproductive failure. Positive $A=$ Simulated positive disease history population under a $10 \%$ annual chance of reproductive failure and a $10 \%$ annual chance of a recurrent adult mass mortality event in exclusive years. Positive $B=$ Simulated positive disease history population under identical conditions to Positive $A$ with addition of a $5 \%$ annual chance of complete recruitment failure and adult mass mortality in the same year. $K=$ Imposed population carrying capacity of 200. 


\section{Discussion}

301 Our results provide the first evidence that a history of ranavirosis in amphibian populations may drive shifts in the age structure of the mature component of host populations, potentially caused by impacts on host life history strategy. We found that breeding populations of $R$. temporaria with a history of ranaviral infection were significantly more likely to contain individuals of $2-5$ years of age than were breeding populations without a known history of exposure to ranavirus. Conversely, disease free populations were more likely to contain older individuals aged 6-10 years old. Our subsequent population matrix modelling revealed that the demographic differences between disease positive and putatively disease free populations might increase the vulnerability of the former to variable

310 environmental conditions that affect recruitment and adult survival. Our data suggest that

311 challenge by disease can mediate shifts in individual life histories that subsequently increase

312 the susceptibility of populations to declines caused by stochastic environmental conditions.

313 This provides a mechanism explaining why, even in the absence of high rates of mortality in 314 younger age classes, disease-challenged populations can suffer higher rates of extirpation 315 over more protracted time scales.

316 The impact of disease on body size and age at sexual maturity

317 Life history theory predicts that when mortality rates in adults are high, reproductive fitness

318 is maximised when reproduction begins earlier in life [46]. We therefore hypothesised that

319 R. temporaria originating from populations where ranavirosis causes increased adult 320 mortality would reach sexual maturity at an earlier age than frogs from disease free 321 populations. We also hypothesised that a trade-off in the allocation of resources to early 322 reproduction rather than growth would cause frogs from disease positive populations to 323 attain a lower body size per age than their counterparts from disease free populations. Both 324 of these hypotheses were also based upon empirical studies conducted on populations of 325 other vertebrates subjected to persistent infectious diseases [1-4]. Surprisingly, we found 
326 that neither of these hypotheses were supported by our data. We found that a positive 327 disease history of ranavirosis does not have a significant impact on either; age at sexual 328 maturity or body size throughout life. This is in contrast with the findings of Scheele et al 329 [47] who did find that an infectious disease $(B d)$ reduced the size and age at sexual maturity of L. v. alpina. The age at which $R$. temporaria mature is intrinsically linked to attaining a minimum body length need to successfully reproduce $[31,35]$. The age at which this length is attained has been shown to be heavily influenced by several environmental factors such as photoperiod and altitude [31]. All frogs in our study were found to mature at either 2, 3 or 4 years 335 of age and this is consistent with the findings of other similar studies on $R$. temporaria $336[31,36,35]$. In fact, no previous study has found male or female $R$. temporaria to reach 337 maturity younger than 2 years of age and it has been shown that post-sexual maturity there 338 is little detectable trade-off between growth and fecundity in response to sub-prime 339 environments [48]. This suggests that the life history strategy of $R$. temporaria may already 340 be optimised to generate maximum reproductive fitness in light of other factors of their 341 environment and that scope for further plasticity in traits such as age at sexual maturity and 342 subsequent growth rates upon the introduction of an infectious disease may be minimal.

\section{The impact of disease on population age structure}

344 Our results illustrate that a history of disease can have a significant effect on the age 345 structure of a population. We found that older $R$. temporaria (aged $6-10$ years) are 346 significantly relatively absent within populations that have a positive history of ranaviral 347 disease. Rana temporaria are highly philopatric [49], and our study populations occupy 348 permanent, urban or semi-urban garden ponds [24,27]. Ranaviruses have been shown to be 349 able to persist in permanent water bodies [41], particularly in the presence of suboptimal or 350 secondary host species $[25,50]$. Mortality due to ranavirosis is annually recurrent $[17,24]$ and 351 unlike many similar host-ranavirus systems globally, infection in the UK seems to primarily 
352 affect adult life stages of $R$. temporaria [22,51]. Apparent adaptive immune response of $R$.

353 temporaria to ranaviruses is limited [52]. It is therefore possible that adult mortality in

354 infected populations is maintained in such a way that an individual is more likely to become

355 infected and die from ranavirosis the more often it returns to spawn in infected water

356 bodies. Such a scenario, would explain the decreased likelihood of observing animals greater

357 than 5 years of age in populations with a positive disease history of ranavirosis.

to be present in populations that have never shown signs of ranavirus infection. Given the pattern, this clear overabundance of young animals in the breeding ponds at disease free populations is more difficult to explain. come in the survival rates of lower age classes [5]. We lack any data on the immature age classes present at our study populations and the snapshot nature of our study means we cannot draw inference on whether the survival rates of younger adult age classes are increased in ranavirus positive populations. However, it is plausible that such an increase could result in the overabundance of younger animals. ranavirus positive populations but not disease free populations suggests that the relatively

371 high number of young animals might be the result of behavioural changes that enhance the 372 life time reproductive success of individuals in the face of high adult mortality. Participation in spawning events in exposed aquatic environments is associated with

374 significant mortality risk to an individual, caused either by exposure to predation or the act 375 of mating itself [44]. Additionally, smaller R. temporaria present in breeding populations are easily out competed by larger individuals, who are more likely to secure a mate and achieve 
378 low is potentially counter-productive to an individual's reproductive fitness meaning that

379 small, less competitive individuals may defer breeding attempts until they are older and

380 larger. However, in environments of high adult mortality the number of lifetime

381 reproductive events is potentially limited. In such environments lifetime, reproductive

382 success is likely to be higher when an individual takes advantage of all possible chances to

383 produce offspring. This may result in smaller, less competitive individuals attempting to

384 breed earlier than they would do in the absence of disease induced mortality.

385 The loss of larger, more competitive individuals at disease positive field sites may

386 also release the smaller individuals from intraspecific competitive pressure. Increased

387 opportunity for less competitive individuals to participate successfully in reproductive

388 events may also result in animals that would normally defer breeding attempts until

389 subsequent years or larger body sizes attempting to reproduce earlier. Any of the above

390 described mechanisms, or a combination of the three would lead to an increase in the

391 number of younger animals in disease positive breeding populations relative to populations

392 that have never experienced disease.

393 The potential impact of disease on the viability of populations

394 When modelled in the absence of environmental variability our representations of both

395 ranavirus positive and disease free populations were able to attain growth rate equilibrium

396 and persist indefinitely. However, the likelihood of extinction increased in simulated positive

397 disease history populations as higher levels of environmental stochasticity were

398 incorporated into our model. These results are consistent with previous observations of age

399 structure truncation increasing the vulnerability of populations to environmental

400 stochasticity $[3,11,53]$.

401 We are afforded a degree of confidence in our population models as the results

402 corroborate previous empirical data collected from the same study system. A long term

403 study of population sizes has shown that following an outbreak of ranavirosis, characterised 
404 by a mass mortality event, UK frog populations follow three possible trajectories. These are:

405 1) complete recovery to post outbreak population levels, 2) persistence at a largely reduced population size, or 3) local extinction [24]. Our simulated $R$. temporaria populations were projected to population sizes that incorporate all of these possible outcomes, dependant on the levels of environmental stochasticity to which a population was subjected.

410 well as in many other species [e.g. 53-57]. The relative absence of the oldest and largest

411 breeding animals from disease positive populations means that per capita fecundity will be 412 reduced and annual recruitment rates lowered. Such changes likely heighten the impacts of 413 any events that result in failed recruitment or further adult mortality.

414 Our simulations suggest that the fate of an infected population may depend heavily 415 upon the environmental conditions in the years following an outbreak, providing a potential 416 explanation of why some populations appear to persist in the presence of an infectious 417 disease, while others are driven to local extirpation. Although we have demonstrated the 418 importance of environmental variation in on the within population disease dynamics of 419 ranavirus, it is not thought to play a role in the wider context of disease spread [27]

\section{The importance of variations in fecundity in population models}

421 It is well known that $R$. temporaria grow continually throughout life $[31,35,48]$, and our data

422 show this to be true in our study populations. We therefore took variations in fecundity by 423 body size into account when constructing our population projection matrix models. In the 424 absence of increased extrinsic mortality, populations simulated by a matrix with varied 425 fecundity were stable and viable. However, when increased adult mortality due to ranaviral 426 exposure was incorporated into our models, the lower fecundity of younger adult age 427 classes played a role in the reduced viability observed. Variations in adult fecundity due to 428 body size are often not included in population projection modelling [eg. 60 but see 61]. 429 However, this result highlights the importance of considering age or body size specific 
430 changes in fecundity in population modelling, particularly when considered threats

431 disproportionately impact certain age classes. Ensuring matrix models of populations 432 represent the life history of the study species as closely as possible is essential, especially 433 when seeking to inform conservation or policy decision making.

\section{Conclusion}

435 Empirical evidence is beginning to accumulate that infectious disease can alter the 436 demographic structure of populations. This is of particular concern in species that are 437 vulnerable to extinction. Our results provide the first evidence that infection with a 438 ranavirus results in age truncation of amphibian populations, despite no obvious change in 439 age or body size at sexual maturity. Population projections showed that the impact of age 440 structure truncation on disease positive populations could potentially be substantial, 441 increasing vulnerability to environmental fluctuations that affect recruitment success. Our 442 results highlight an increasing need to better understand the impact of disease on life 443 history and the demography of host populations. Further investigation of this relationship, 444 possibly via a long-term mark-recapture study on the same populations used here could 445 elucidate the exact mechanisms by which these demographic shifts are generated. This work 446 provides evidence that the emergence of an infectious disease within a population can 447 heighten its vulnerability to external stressors. Although in this case our theoretical stressors 448 were environmental in origin the same is likely to be true for all types of stressor, including 449 anthropogenic.

\section{Ethics statement}

451 This project was approved by the ethics boards of both the University of Exeter and

452 Zoological Society of London and conducted under the project license $80 / 2466$ issued by the 453 UK Home Office. All field sampling was conducted under the personal Home Office license $454 \quad 30 / 10730$ issued to Lewis Campbell. 
The raw data file and $\mathrm{R}$ scripts used in the analyses of this work are available online via

GitHub at the following link;

https://github.com/zoolew/Ranavirus-FrogDemography

\section{Author contributions}

461 LJC devised the study, collected field samples, prepared samples in the laboratory, analysed

data and wrote the manuscript. GT performed skeletochronology and provided editorial

\section{Competing interests}

\section{Funding}

471 This work was funded by a Natural Environment Research Council PhD studentship held by

LJC.

\section{References}

1. Lachish S, McCallum H, Jones M. Demography, disease and the devil: Life-history changes in a disease-affected population of Tasmanian devils (Sarcophilus harrisii). J Anim Ecol. 2009;78: 427-436. doi:10.1111/j.1365-2656.2008.01494.x

2. Jones ME, Cockburn A, Hamede R, Hawkins C, Hesterman H, Lachish S, et al. Lifehistory change in disease-ravaged Tasmanian devil populations. Proc Natl Acad Sci U S A. 2008;105: 10023-10027. doi:10.1073/pnas.0711236105

3. Ohlberger J, Langangen $\emptyset$, Edeline E, Moland Olsen E, Winfield IJ, Fletcher JM, et al. Pathogen-induced rapid evolution in a vertebrate life-history trait. Vøllestad

4. Fitzpatrick SW, Torres-Dowdall J, Reznick DN, Ghalambor CK, Chris Funk W. Parallelism Isn't Perfect: Could Disease and Flooding Drive a Life-History 
Anomaly in Trinidadian Guppies? Am Nat. 2014;183: 290-300. doi:10.1086/674611

5. Stearns SC. The evolution of life histories. Oxford University Press; 1992.

6. Roff DA. The evolution of life histories : theory and analysis. Chapman \& Hall; 1993.

7. Stearns SC, Ackermann M, Doebeli M, Kaiser M. Experimental evolution of aging, growth, and reproduction in fruitflies. Proc Natl Acad Sci. 2000;97: 3309-3313. doi:10.1073/pnas.97.7.3309

8. Reznick DA, Bryga H, Endler JA. Experimentally induced life-history evolution in a natural population. Nature. 1990;346: 357-359. doi:10.1038/346183a0

9. Olsen EM, Heino M, Lilly GR, Morgan MJJ, Brattey J, Ernande B, et al. Maturation trends indicative of rapid evolution preceded the collapse of northern cod. Nature. 2004;428: 932-935. doi:10.1038/nature02453.1.

10. Saether B-E, Bakke O. Avian Life History Variation and Contribution of Demographic Traits to the Population Growth Rate. Ecology. 2000;81: 642-653.

11. Scheele BC, Hunter DA, Banks SC, Pierson JC, Skerratt LF, Webb R, et al. High adult mortality in disease-challenged frog populations increases vulnerability to drought. J Anim Ecol. 2016; 1-8. doi:10.1111/1365-2656.12569

12. Jones KE, Patel NG, Levy MA. Global trends in emerging infectious diseases. Nature. 2008;451: 990-993. doi:10.1038/nature06536

13. Wake DB, Vredenburg VT. Are we in the midst of the sixth mass extinction? A view from the world of amphibians. Proc Natl Acad Sci U S A. 2008;105: 1146611473. doi:10.1073/pnas.0801921105

14. Cushman SA. Effects of habitat loss and fragmentation on amphibians: A review and prospectus. Biol Conserv. 2006;128: 231-240. doi:10.1016/j.biocon.2005.09.031

15. Xie F, Lau MWN, Stuart SN, Chanson JS, Cox NA, Fischman DL. Conservation needs of amphibians in China: A review. Sci China, Ser C Life Sci. 2007;50: 265-276. doi:10.1007/s11427-007-0021-5

16. Foden WB, Butchart SHM, Stuart SN, Vié JC, Akçakaya HR, Angulo A, et al. Identifying the World's Most Climate Change Vulnerable Species: A Systematic Trait-Based Assessment of all Birds, Amphibians and Corals. PLoS One. 2013;8. doi:10.1371/journal.pone.0065427

17. Daszak P, Berger L, Cunningham A a, Hyatt a D, Green DE, Speare R. Emerging infectious diseases and amphibian population declines. Emerg Infect Dis. 1999;5: 735-48. doi:10.3201/eid0506.990601

18. Chinchar VG. Ranaviruses (family Iridoviridae): Emerging cold-blooded killers. Arch Virol. 2002;147: 447-470. doi:10.1007/s007050200000

19. Green DE, Converse K a, Schrader AK. Epizootiology of sixty-four amphibian morbidity and mortality events in the USA, 1996-2001. Ann N Y Acad Sci. 2002;969: 323-339. doi:10.1111/j.1749-6632.2002.tb04400.x

20. Whittington RJ, Becker J a, Dennis MM. Iridovirus infections in finfish - critical review with emphasis on ranaviruses. J Fish Dis. 2010;33: 95-122. doi:10.1111/j.1365-2761.2009.01110.x

21. Marschang RE. Viruses infecting reptiles. Viruses. 2011;3: 2087-2126. doi: $10.3390 / \mathrm{v} 3112087$

22. Cunningham a a, Langton TE, Bennett PM, Lewin JF, Drury SE, Gough RE, et al. Pathological and microbiological findings from incidents of unusual mortality of the common frog (Rana temporaria). Philos Trans R Soc Lond B Biol Sci. 1996;351: 1539-1557. doi:10.1098/rstb.1996.0140

23. Bayley AE, Hill BJ, Feist SW. Susceptibility of the European common frog Rana temporaria to a panel of ranavirus isolates from fish and amphibian hosts. Dis Aquat Organ. 2013;103: 171-183. doi:10.3354/dao02574

24. Teacher a.GF, Cunningham a. a., Garner TWJ. Assessing the long-term impact of 
Ranavirus infection in wild common frog populations. Anim Conserv. 2010;13: 514-522. doi:10.1111/j.1469-1795.2010.00373.x

25. Hoverman JT, Gray MJ, Haislip NA, Miller DL. Phylogeny, life history, and ecology contribute to differences in amphibian susceptibility to ranaviruses. Ecohealth. 2011;8: 301-319. doi:10.1007/s10393-011-0717-7

26. Warne RW, Crespi EJ, Brunner JL. Escape from the pond: Stress and developmental responses to ranavirus infection in wood frog tadpoles. Funct Ecol. 2011;25: 139-146. doi:10.1111/j.1365-2435.2010.01793.x

27. Price S, Garner T, Cunningham A, Langton T, Nichols R. Reconstructing the emergence of a lethal infectious disease of wildlife supports a key role for spread through translocations by humans. Proc R Soc B. 2016;283: 20160952. doi:10.1098/rspb.2016.0952

28. Berven KA. Factors affecting population fluctuations in larval and adult stages of the wood frog (Rana sylvatica). Ecology. 1990;71: 1599-1608. doi:10.2307/1938295

29. Wilbur HM. Complex Life Cycles. Annu Rev Ecol Syst. 1980;11: 67-93.

30. Verrell PA. Return to water by juvenile amphibians at a pond in southern England. Amphibia-Reptilia. 1985;6: 93-96.

31. Miaud C, Guyétant R, Elmberg J. Variations in life-history traits in the common frog Rana temporaria (Amphibia: Anura): a literature review and new data from the French Alps. J Zool. 1999;249: 61-73. doi:10.1017/S0952836999009061

32. Sinsch U. Life-History Traits in Amphibians. Herpetol J. 2015;25: 5-13.

33. Team R. R: A language and environment for statistical computing. R Foundation for Statistical Computing, Vienna, Austria. 2013. 2014;

34. Bates D, Maechler M, Bolker B, Walker S. Fitting Linear Mixed-Effects Models Using lme4. J Stat Softw. 2015;67: 1-48. doi:10.18637/jss.v067.i01.

35. Ryser J. Comparative life histories of a low and high-elevation population of the common frog Rana temporaria. Amphibia-Reptilia. 1996;27: 183-195.

36. Gibbons MM. Reproduction, growth and demography of frogs, Rana temporaria, in the west of Ireland. University, College, Galway. 1983.

37. Hadfield JD. MCMC methods for multi-response generalized linear mixed models: the MCMCglmm R package. J Stat Softw. 2010;33: 1-22. doi:10.1002/ana.22635

38. Biek R, Funk WC, Maxell B a, Mills LS. What Is Missing from Insights Amphibian Decline Ecological Sensitivity Analysis. Conserv Biol. 2002;16: 728-734. doi:10.1046/j.1523-1739.2002.00433.x

39. Gibbons MM, McCarthy TK. The reproductive output of frogs Rana temporaria (L.) with particular reference to body size and age. J Zool. 1986;209: 579-593. Available: http://onlinelibrary.wiley.com/doi/10.1111/j.14697998.1986.tb03613.x/abstract

40. Stott I, Hodgson DJ, Townley S. Popdemo: provides tools for demographic modelling using project matrices. 2012.

41. Nazir J, Spengler M, Marschang RE. Environmental persistence of amphibian and reptilian ranaviruses. 2012;98: 177-184. doi:10.3354/dao02443

42. Shoo LP, Olson DH, Mcmenamin SK, Murray KA, Van Sluys M, Donnelly MA, et al. Engineering a future for amphibians under climate change. J Appl Ecol. 2011;48: 487-492. doi:10.1111/j.1365-2664.2010.01942.x

43. Beattie RC, Aston RJ, Milner AGP. A field study of fertilization and embryonic development in the common frog (Rana temporaria) with particular reference to acidity and temperature. J Appl Ecol. 1991;28: 346-357. doi:10.2307/2404134

44. Beebee TJC (Trevor JC. Ecology and conservation of amphibians [Internet]. Chapman \& Hall; 1996. Available: http://www.springer.com/gb/book/9780412624100

45. Stubben C, Milligan B. Estimating and analyzing demographic models. J Stat Softw. 2007;22: 1-23. doi:10.18637/jss.v022.i11 
46. Agnew P, C. Koella J, Michalakis Y. Host life history responses to parasitism. Microbes Infect. 2000;2: 891-896. doi:10.1016/S1286-4579(00)00389-0

47. Scheele BC, Skerratt LF, Hunter DA, Banks SC, Pierson JC, Driscoll DA, et al. Disease-associated change in an amphibian life-history trait. Oecologia. 2017; doi:10.1007/s00442-017-3911-7

48. Lardner B, Loman J. Growth or reproduction ? Resource allocation by female frogs Rana temporaria. 2003; 541-546. doi:10.1007/s00442-003-1390-5

49. Brabec M, Czech T, Bocek R, Ecology RB. Spring migration distances of some Central European amphibian species Spring migration distances of some Central European. 2009; doi:10.1163/156853809788795236

50. North AC, Hodgson DJ, Price SJ, Griffiths AGF. Anthropogenic and Ecological Drivers of Amphibian Disease (Ranavirosis). PLoS One. 2015;10: e0127037. doi:10.1371/journal.pone.0127037

51. Duffus ALJ, Nichols RA, Garner TWJ. Investigations into the life history stages of the common frog (Rana temporaria) affected by an amphibian ranavirus in the United Kingdom. Herpetol Rev. 2013;44: 260-263.

52. Price SJ, Garner TWJ, Balloux F, Ruis C, Paszkiewicz KH, Moore K, et al. A de novo Assembly of the Common Frog (Rana temporaria) Transcriptome and Comparison of Transcription Following Exposure to Ranavirus and Batrachochytrium dendrobatidis. PLoS One. 2015;10: e0130500. doi:10.1371/journal.pone.0130500

53. Rouyer T, Sadykov A, Ohlberger J, Stenseth NC. Does increasing mortality change the response of fish populations to environmental fluctuations? Ecol Lett. 2012;15: 658-665. doi:10.1111/j.1461-0248.2012.01781.x

54. Blueweiss L, Fox H, Kudzma V, Nakashima D, Peters R, Sams S. Relationships between body size and some life history parameters. Oecologia. 1978;37: 257272. doi: $10.1007 /$ BF00344996

55. Trippel EA. Relations of Fecundity, Maturation, and Body Size of Lake Trout, and Implications for Management in Northwestern Ontario Lakes. North Am J Fish Manag. 1993;5947. doi:10.1577/1548-8675(1993)013<0064

56. Sand H. Life history patterns in female moose (Alces alces): the relationship between age, body size, fecundity and environmental conditions. Oecologia. 1996;106: 212-220. doi:10.1007/BF00328601

57. Honěk A. Intraspecific Variation in Body Size and Fecundity in Insects : A General Relationship. 1993;66: 483-492.

58. Penteriani V, Balbontin J, Ferrer M. Simultaneous effects of age and territory quality on fecundity in Bonelli 's Eagle Hieraaetus fasciatus. 2003;145: 77-82.

59. Briggs CJ, Vredenburg VT, Knapp RA, Rachowicz LJ. Investigating the PopulationLevel Effects of Chytridiomycosis : An Emerging Infectious Disease of Amphibians. Ecology. 2005;86: 3149-3159.

60. Zambrano L, Vega E, Herrera MLG, Prado E, Reynoso VH. A population matrix model and population viability analysis to predict the fate of endangered species in highly managed water systems. Anim Conserv. 2007;10: 297-303. doi:10.1111/j.1469-1795.2007.00105.x 